\title{
Liposome encapsulated of temozolomide for the treatment of glioma tumor: preparation, characterization and evaluation
}

\author{
Jinhua Gao ${ }^{1}$, Zhonglan Wang ${ }^{1}$, Honghai Liu ${ }^{2}$, Longmei Wang ${ }^{1}$, Guihua Huang ${ }^{1, *}$ \\ ${ }^{1}$ School of Pharmaceutical Science, Shandong University, Ji'nan, Shandong, China; \\ ${ }^{2}$ Food and Drug Administration of Dezhou, Dezhou, Shandong, China.
}

\begin{abstract}
Summary
Temozolomide plays a critical role in curing glioma at present. The purpose of this work was to develop a suitable drug delivery system which could prolong the half-life, improve the brain targeting, and reduce the systemic effect of the drug. Temozolomide-liposomes were formulated by the method of proliposomes. They were found to be relatively uniform in size of $156.70 \pm 11.40 \mathrm{~nm}$ with a narrow polydispersity index (PI) of $0.29 \pm 0.04$. The average drug entrapment efficiency and loading capacity were $35.45 \pm 1.48 \%$ and $2.81 \pm$ $0.20 \%$, respectively. The $\mathrm{pH}$ of temozolomide-liposomes was 6.46 . In vitro release studies were conducted by a dynamic dialysis. The results showed that temozolomide released slowly from liposomes compared with the solution group. The release behavior of temozolomideliposomes was in line with First-order kinetics and Weibull equation. The pharmacokinetics study was evaluated by pharmacokinetics parameters. The $t_{1 / 2 \beta}$ and MRT of temozolomideliposomes were 3.57 times and 1.27 times greater than that of temozolomide solution. The $\mathrm{C}_{\max }$ and AUC values of temozolomide-liposomes were 1.10 times and 1.55 times greater than that of temozolomide solution. The results of pharmacokinetics study showed temozolomideliposomes prolonged the in vivo circulation time and increased AUC. Furthermore, the biodistribution in mice showed that temozolomide-liposomes preferentially decreased the accumulation of temozolomide in heart and lung and increased the drug concentration in brain after i.v. injection, which implied that temozolomide-liposomes improved the therapeutic effect in the brain and reduced the toxicity in lung and heart.
\end{abstract}

Keywords: Temozolomide, liposomes, gliomas, pharmacokinetics, biodistribution

\section{Introduction}

Glioma with an incidence of 5 per 100,000 persons is one of the most devitalizing malignant diseases (1). They are very difficult to resect entirely because of the infiltrative nature of the tumors and these tumors almost invariably recur, rapidly leading to death (2). Surgical resection alone is inadequate for cure, so enough effective chemotherapy drugs are desiderated.

Temozolomide is a new type of imidazole tetrazines drugs with demonstrated efficacy for patients with recurrent gliomas. During adjuvant temozolomide, the median survival of the patient with gliomas was about 16

*Address correspondence to:

Dr. Guihua Huang, School of Pharmaceutical Science, Shandong University, 44 Wenhua Xi Road, Ji'nan, Shandong, China.

E-mail: hgh2003@gmail.com months, and the survival rates of 1-year and 2-year were $58 \%$ and $31 \%$, respectively (3). Generally, temozolomide is regarded as tolerable, effective, and well absorbed after oral administration. However, because of its short half-life of about $1.8 \mathrm{~h}$ in plasma, temozolomide must be administered in high systemic doses to achieve therapeutic brain levels (4). Furthermore, prolonged systemic administration is associated with systemic effect including nausea and vomiting, headache, and fatigue (5). Temozolomide injection is more suitable for post-operative patients. However, the marketable temozolomide lyophilized powder for injection used Tween 80 due to the poor solubility of the drug, which can cause hemolysis, allergies and other adverse reactions $(6,7)$. Therefore, the development of new anti-glioma nano drug delivery systems became the research focuses to prolong the half-life, improve the brain targeting, and reduce the systemic effect of temozolomide. Huang et al. (8) prepared the solid lipid nanoparticles of 
temozolomide, which exhibited sustained release and brain targeting.

Liposomes as the first closed bilayer phospholipid systems were described in 1965 and they were proposed as drug delivery systems soon (9). Both hydrophilic drugs and hydrophobic drug can be entrapped. The significant advance was the ability to keep the integrity of the liposome structure when entrapping drugs in it. This property has been demonstrated to significantly affect the stability and pharmacokinetics of dugs (10). With this property liposomes can protect temozolomide from depredating in the plasma, which can improve the stability of the temozolomide and prolong considerably the temozolomide half-life in the circulation. In addition, liposome could be consumed by monocytes, neutrophils, etc., which can pass through blood brain barrier in the body circulation process selectively and get to the brain (11). Besides, it can get to the brain tissue through the blood brain barrier by pinocytosis of endothelial cells $(12,13)$. So the drug concentration in the brain could increase significantly, when the temozolomide was entrapped in liposome.

The aim of this present work was to develop temozolomide-liposomes to prolong the half-life, reduce the systemic effect, and improve the brain targeting, the therapeutic efficacy and the patient compliance. In this study, temozolomide-liposomes was developed by the method of proliposomes method with the materials of soybean lecithin and cholesterol and characterized by the particle size and its distribution, $\mathrm{pH}$ value, morphology, drug loading, drug encapsulation efficiency, and drug release behavior in vitro. The in vivo pharmacokinetics and biodistribution of temozolomide-liposomes were evaluated to elucidate their feasibility as intravenous delivery systems.

\section{Materials and Methods}

\subsection{Materials}

Temozolomide was purchased from Hangzhou Hesu Chemical Technology Co., Ltd. (Zhejiang, China). Soybean lecithin (injection grade, phosphatidylcholine accounts for $95 \% \mathrm{pH} 5.0-7.0$ ) was provided by Shanghai Taiwei Pharmaceutical Co., Ltd. (Shanghai, China). Cholesterol, Sorbitol, and Mannitol were purchased from Sinopharm Chemical Reagent Co., Ltd. (Beijing, China). Lactin was provided by Tianjin Guangcheng Chemical Agent Co., Ltd. (Tianjin, China). Glacial acetic acid was purchased from Tianjin Fuyu Chemical Co., Ltd. (Tianjin, China). N-caprylic alcohol, hydrochloric acid, and other reagents used were of analytical grade.

\subsection{Formulation of temozolomide-liposomes}

Temozolomide-liposome was prepared by a proliposomes method $(14,15)$. Briefly, temozolomide (20 $\mathrm{mg}$ ), soybean phospholipid (240 mg), and cholesterol (30 $\mathrm{mg})$ were dissolved in methanol. Then sorbitol $(1.5 \mathrm{~g})$ was added with the help of ultrasonic cleaner (Kun Shan Ultrasonic Instruments Co., Ltd., Jiangsu, China). The organic solvent was removed using a RE52-98 rotary evaporator (Shanghai Yarong Instrument Co., Shanghai, China) under vacuum $(0.02 \mathrm{MPa})$ with the rotate speed of $80 \mathrm{rpm}$ for $30 \mathrm{~min}$ at $40^{\circ} \mathrm{C}$. The lipid was thoroughly dried to remove residual organic solvent by placing in a vacuum oven for $5 \mathrm{~h}$ and then filtered through the screen (20 mesh). All products were sealed immediately in vials and stored in refrigerator at $4^{\circ} \mathrm{C}$. The dried lipid was rehydrated with $15 \mathrm{~mL}$ of the solution of temozolomide (1 $\mathrm{mg} / \mathrm{mL}$ ) and obtained suspensions with ultrasound for 5 min when use.

\subsection{Measurement of particle size, and $\mathrm{pH}$ value}

The particle size and polydispersity index (PI) of temozolomide-liposome were determined by Delsa ${ }^{\mathrm{TM}}$ Nano C Particle Analyzer (Beckman Coulter A53878, Otsuka Blectronics Co. Ltd., USA). The $\mathrm{pH}$ value of temozolomide-liposome was determined with a digital pH meter (FE20, Mettler Toledo, Switzerland). Each measurement was made at least in triplicate

\subsection{Transmission election microscope (TEM) examination}

TEM observations of temozolomide-liposomes were performed by transmission electron microscope $(\mathrm{H}-$ 7000, Hitachi, Japan). Briefly, the liposome samples were dropped onto carbon-coated grids and drawn off excess solution with a piece of filter paper. Then, the grids were immersed in $2.0 \%$ phosphomolybdic acid aqueous solution for $1 \mathrm{~min}$. Finally, the grids were dried and imaged using a transmission electron microscope.

\subsection{Determination of entrapment efficiency (EE) and drug-loading rate}

The EE of liposomes was determined using the ultrafiltration technique (molecular weight cutoff $10 \mathrm{kDa}$ ) for separating the non-entrapped drug from liposomes (16). In short, $50 \mu \mathrm{L}$ of temozolomide-liposome was mixed with $1 \mathrm{~mL}$ of acetonitrile, and diluted it to $10 \mathrm{~mL}$ with $5 \%$ acetic acid (v/v). The samples were filtered using $0.22 \mu \mathrm{m}$ membrane filter and then subjected to high-performance liquid chromatography (HPLC). The content of total temozolomide was determined.

Briefly, $100 \mu \mathrm{L}$ of temozolomide liposome was added in an ultrafiltration centrifuge tube and mixed with $700 \mu \mathrm{L}$ of distilled water. The free temozolomide was separated from liposome by ultrafiltration at 3,500 $\mathrm{rpm}$ for $30 \mathrm{~min}$. After completed the centrifugation, $200 \mu \mathrm{L}$ of distilled water was added and shake, then centrifuge again by ultracentrifugation at 3,500 rpm 
for 20 min. Suck up $50 \mu \mathrm{L}$ of filtrate and dilute to $10 \mathrm{~mL}$ with $5 \%$ acetic acid (v/v). The samples were filtered using $0.22 \mu \mathrm{m}$ membrane filter and injected into the HPLC. The content of free temozolomide was determined.

$$
\begin{aligned}
& E E \%=\frac{W_{\text {total }}-W_{\text {free }}}{W_{\text {total }}} \times 100 \% \\
& D L \%=\frac{W_{\text {total }}-W_{\text {free }}}{W_{\text {lipid }}} \times 100 \%
\end{aligned}
$$

Where $W_{\text {free }}$ was the analyzed weight of free drug, $W_{\text {total }}$ was the analyzed weight of drug in liposomes, $W_{\text {lipid }}$ was the analyzed weight of liposomes.

\subsection{HPLC analysis}

The HPLC equipment was a Shimadzu LC-10A system (Shimadzu, Kyoto, Japan) consisting of a LC-10AT HPLC pump and a SPD-10A UV-VIS detector. Data processing was performed with LC Solution software. Drug analysis was conducted on a Diamonsil-ODS column $(150 \times 4.6 \mathrm{~mm}$, Dikma Technologues, China) . The mobile phase consisted of methanol, deionized water, and glacial acetic acid (10:89.5:0.5, v/v) at a flow rate of $1.0 \mathrm{~mL} / \mathrm{min}$ and an injection volume was $20 \mathrm{~mL}$. The detection wavelength was set at $329 \mathrm{~nm}$

\subsection{In vitro release study}

In vitro release rate behaviors of temozolomide from liposome vesicles were performed by the dialysis method. Briefly, $3 \mathrm{~mL}$ of liposome suspension (the concentration is $1 \mathrm{mg} / \mathrm{mL}$ ) was loaded in dialysis bag (molecular weight cutoff 8 to $14 \mathrm{kDa}$ ) and dialyzed against the release media of PBS with $\mathrm{pH} 5.0$ at $37 \pm 0.5^{\circ} \mathrm{C}$ in an water bath shaker at $100 \mathrm{rpm}$. At scheduled time $(0.083,0.17,0.33,0.5,0.75,1,2,4$, and $6 \mathrm{~h})$ intervals, $0.2 \mathrm{~mL}$ of the release media was collected and then the same volume of fresh release media were added. Dilute the solution to $1 \mathrm{~mL}$ with $5 \%$ acetic acid (v/v). The release amount of temozolomide was determined by HPLC as described earlier. The mean calculated values were obtained from 3 replicates. The drug release profile of temozolomide was examined as a control. The accumulative release percentage of temozolomide (R\%) was calculated according to the following equation:

$$
R=\frac{c_{n} v_{0}+\sum_{i=0}^{n-1} c_{i} v_{i}}{W} \times 100 \%
$$

where $R$ is the release rate, $c_{n}$ is the drug concentration in the release medium of each time interval, $v_{0}$ is the total volume of the release medium, $v_{i}$ is the volume of the withdrawn medium, $c_{i}$ is the drug concentration in the release medium at time, and $\mathrm{W}$ is the total drug content of the release sample.

\subsection{Pharmacokinetics studies in rabbits}

The in vivo pharmacokinetics experiment was carried out with adult white New Zealand rabbits (female and male) weighing 2.0-2.5 $\mathrm{kg}$ supplied by the Medical Animal Test Center of Shandong University. The animal experiment protocol was reviewed and approved by the Institutional Animal Care and Use Committee of Shandong University. The rabbits used for this study were housed individually under normal conditions, and fasted overnight before experiment with free access to water. Animals were randomly assigned into two groups with six rabbits each. Rabbits in one group were administered with temozolomide liposomes (equal to $10 \mathrm{mg} / \mathrm{kg}$ temozolomide) by injection in one of the ear marginal vein, while control group was administered with $10 \mathrm{mg} / \mathrm{kg}$ temozolomide solution $(5 \mathrm{mg} / \mathrm{mL}$, dissolved in physiological saline solution). Blood was collected at specified time intervals $(0.083,0.167,0.333$, $0.5,1,2,3,5,8,10,12 \mathrm{~h}$ ) by intracardiac puncture and was put into centrifuge tube washed with EDTA-2Na (15 mg/mL) along with $10 \mu \mathrm{L}$ of a $10 \%$ phosphoric acid solution. Plasma samples were harvested by centrifuging at $12,000 \mathrm{rpm}$ for $10 \mathrm{~min}$. Subsequently, $300 \mu \mathrm{L}$ of the resulting rabbit plasma was added to $300 \mu \mathrm{L}$ protein precipitation $(10 \%$ trichloroacetic acid:acetonitrile $=3: 2$ ) and the mixture was vortexed for $30 \mathrm{sec}$. The sample was centrifuged for $10 \mathrm{~min}$ at 12,000 rpm. After centrifugation, the supernatant layer was filtered through a $0.22 \mu \mathrm{m}$ filter, and then $20 \mu \mathrm{L}$ of the filtered solution was directly injected into HPLC for analysis.

The plasma samples were determined by the HPLC system. The HPLC condition was the same as described above except for the mobile phase consisted of methanol, deionized water, and glacial acetic acid $(8: 91.5 .5: 0.5, \mathrm{v} / \mathrm{v})$.

Method validation: weigh $61.2 \mathrm{mg}$ of temozolomide, then dissolve and dilute to $50 \mathrm{~mL}$ with water. Put the temozolomide solution as stock solution. Pipe appropriate amount stock solution to prepare a series of concentration of temozolomide $(1.5,3,30,150,306,612$, $1,224 \mu \mathrm{g} / \mathrm{mL}$ ). Pipe $10 \mu \mathrm{L}$ of the series of concentration of temozolomide solution respectively, then add 0.28 $\mathrm{mL}$ of plasma and $10 \mu \mathrm{L}$ of $10 \%$ phosphoric acid solution $(\mathrm{w} / \mathrm{v})$, mix well. Then a series of concentration of temozolomide $(0.051,0.1,1.02,5.1,10.2,20.4$, $40.8 \mu \mathrm{g} / \mathrm{mL}$ ) were obtained. Dispose the plasma with temozolomide with the method as above. Injected $20 \mu \mathrm{L}$ sample and record the peak area (A). Draw the standard curve and calculate the $\mathrm{CV} \%$ and recovery.

\subsection{Drug distribution studies in mice}

Kunming strain mice (weighed between 18 and $22 \mathrm{~g}$, female or male were provided by the Medical Animal Test Center of Shandong University) were used for the biodistribution studies. The animals were fasted $12 \mathrm{~h}$ 
before drug administration. The animal experiment protocol was reviewed and approved by the Institutional Animal Care and Use Committee of Shandong University.

Two groups of 15 mice each were used for the in vivo distribution studies. temozolomide-liposomes and temozolomide solution were injected through the tail veins of mice at a dosage of $20 \mathrm{mg} / \mathrm{kg}$. Following drug administration, at each predetermined time point $(0.083,0.25,0.5,1,2 \mathrm{~h})$, three mice in each group were then sacrificed by cervical dislocation, and the spleen, kidneys, liver, brain, heart, and lungs were surgically removed. Each organ sample was washed with physiological saline solution, and the redundant liquid was blotted using absorbent paper. Accurately weighted tissue specimen was homogenized with $1 \mathrm{~mL}$ physiological saline solution (except for liver, $2 \mathrm{~mL}$ ) by homogenizer. Tissue homogenates were processed as the plasma sample. After centrifugation, the supernatant was passed through a $0.22 \mu \mathrm{m}$ filter. About $20 \mu \mathrm{L}$ of the filtered tissue homogenate was injected into HPLC for analysis. The HPLC condition was the same as described in Pharmacokinetics studies in rabbits.

\section{Results and Discussions}

\subsection{Physicochemical characterization of the liposome}

The photographs of three batches of the formulation were shown in Figure 1. The appearance of liposomes after rehydration is semitransparent with opalescence.

The particle size, polydispersity index, entrapment efficiency, drug loading, and $\mathrm{pH}$ of three batches of

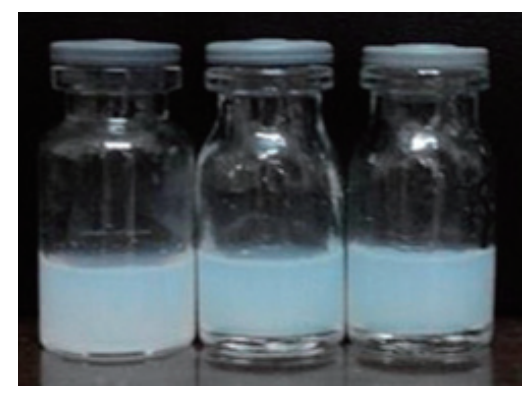

Figure 1. Appearance of liposomes after rehydration. The photographs of three batches of the formulation were shown. The appearance of liposomes after rehydration is semitransparent with opalescence. the formulation were reported in Table 1. The mean particle size of the formulation was $156.7 \pm 11.4 \mathrm{~nm}$. Furthermore, the PI of temozolomide-liposomes was $0.290 \pm 0.041$, which revealed that the formulation had a quite narrow size distribution and uniform size. The mean $\mathrm{pH}$ values of temozolomide-liposomes was $6.46 \pm 0.08$, which was within the safe $\mathrm{pH}$ scope of 4-9 for intravenous injection. The mean drug entrapment efficiency and drug loading was $35.45 \pm 1.48 \%$ and $2.81 \pm 0.20 \%$, respectively. The size distribution of temozolomide-liposomes shown in Figure 2. The results indicated that singe-peak and narrow distribution were obtained.

To obtain more information about the morphology of temozolomide-liposomes, TEM analysis was performed and the result of TEM image was showed in Figure 3. The image displayed that temozolomideliposomes had relatively spherical shapes and had obvious hydrophilic layer, which may be due to sorbitol covering in the surface of the liposome. The particle

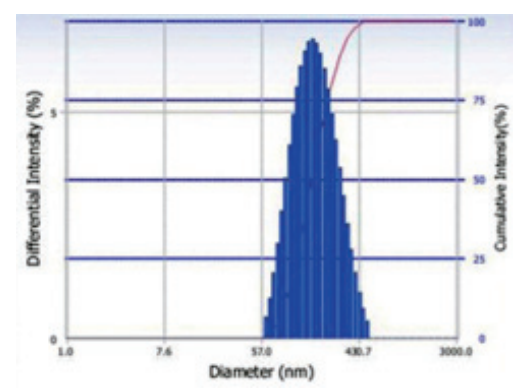

Figure 2. The size distribution of temozolomide liposomes. The results indicated that singe-peak and narrow distribution were obtained.

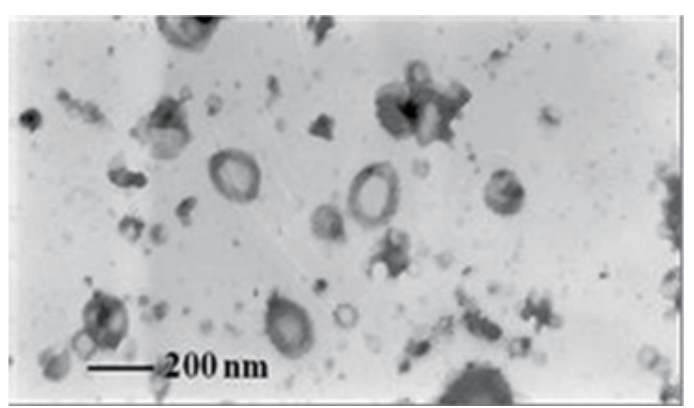

Figure 3. Transmission electron microscopy micrographs of temozolomide. The image displayed that temozolomideliposomes had relatively spherical shapes and had obvious hydrophilic layer, which may be due to sorbitol covering in the surface of the liposome.

Table 1. The particle size, polydispersity index, entrapment efficiency, drug loading and pH of three batches of the formulation

\begin{tabular}{|c|c|c|c|c|c|}
\hline Batches & Particle size (nm) & PI & $\mathrm{EE}(\%)$ & DL $(\%)$ & $\mathrm{pH}$ \\
\hline 1 & 159.4 & 0.334 & 36.40 & 2.70 & 6.49 \\
\hline 2 & 144.2 & 0.282 & 33.75 & 2.68 & 6.53 \\
\hline 3 & 166.5 & 0.254 & 36.21 & 3.04 & 6.37 \\
\hline $\bar{\chi} \pm$ S.D. & $156.7 \pm 11.4$ & $0.290 \pm 0.041$ & $35.45 \pm 1.48$ & $2.81 \pm 0.20$ & $6.46 \pm 0.08$ \\
\hline
\end{tabular}

PI, polydispersity index; EE, entrapment efficiency; DL, drug loading. 


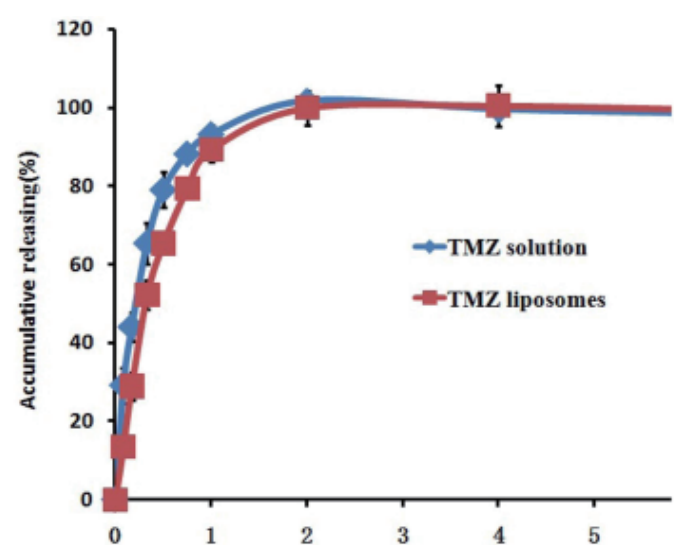

Figure 4. In vitro release profile of temozolomide from temozolomide solution and temozolomide-liposomes in phosphate-buffered saline $\left(\mathrm{pH} \mathrm{5.0)}\right.$ at $37 \pm 0.5^{\circ} \mathrm{C}(n=3)$. The release behavior of temozolomide- liposomes was in accordance with the Weibull equation and First-order kinetics.

size determined by TEM agreed well with the results from Delsa ${ }^{\mathrm{TM}}$ Nano CParticle Analyzer.

\subsection{In vitro release behaviors of temozolomide- liposomes}

The release behavior profile of temozolomide-liposomes and temozolomide solution in vitro was shown in Figure 4. The release behavior of temozolomide-liposomes was in accordance with the Weibull equation and Firstorder kinetics. It can be expressed using the following equation: $\ln \ln (1 /(1-\mathrm{Q} / 100))=1.0805 \ln t+0.8133$, $r=0.9988$ and $\ln (100-\mathrm{Q})=-2.2329 \mathrm{t}+4.6426, r=$ 0.9988 . The release behavior of temozolomide solution was in accordance with the Weibull equation model and can be expressed using the following equation: $\ln \ln (1 /$ $(1-\mathrm{Q} / 100))=0.84191 \mathrm{nt}+1.0014, r=0.9992$. The results of in vitro release profile of temozolomide from temozolomide solution and temozolomide-liposomes were shown that temozolomide was released slower from temozolomide-liposomes than temozolomide solution at first. The slow release characteristic at this phase could be attributed to the fact that temozolomide was held by the lipid and therefore temozolomide was released gradually from the lipid matrices mainly through dissolution and diffusion (17). However, after $2 \mathrm{~h}$, the release rate of the drug from the liposomes and solution were both observed around $100 \%$ and their release behaviors have no obvious difference. This phenomenon may be explained by the existing of the free drugs in the preparations because of the low encapsulation efficiency.

\subsection{Pharmacokinetics study in rabbits}

At the range of $0.051-40.8 \mu \mathrm{g} / \mathrm{mL}$, the standard curve was $\mathrm{A}=32.423 \mathrm{C}-2.6433$ and the standard showed a good linearity with a correlation coefficient of 0.9999 . The $\mathrm{CV} \%$ of is no more than $6 \%$ and the recovery is no

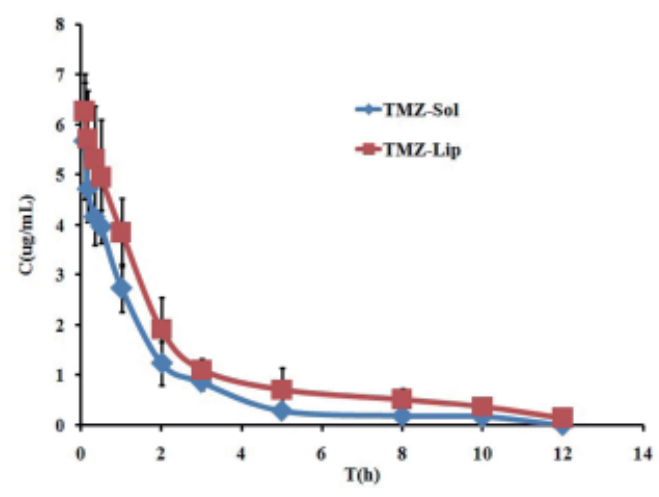

Figure 5. The temozolomide concentration-time curve after intravenous administration of temozolomide solution and temozolomide- liposomes in rabbits $(\boldsymbol{n}=\mathbf{6})$. The plasma temozolomide concentration of temozolomide-liposomes group was much higher than that of the temozolomide solution group at each sampling time.

Table 2. The pharmacokinetic parameters of temozolomide after $i$.v. injection of temozolomide solution and temozolomide-liposomes in rabbits $(n=6)$

\begin{tabular}{lcc}
\hline Parameters & Temozolomide & Temozolomide \\
\hline & -Solution & -Liposomes \\
$\mathrm{t} 1 / 2 \alpha(\mathrm{h})$ & 0.745 & \\
$\mathrm{t} 1 / 2 \beta(\mathrm{h})$ & 3.731 & 1.050 \\
$\mathrm{~V} 1(\mathrm{~L} / \mathrm{kg})$ & 1.741 & 13.312 \\
$\mathrm{CL}(\mathrm{L} / \mathrm{h} / \mathrm{kg})$ & 0.956 & 0.631 \\
$\mathrm{AUC}-\mathrm{t}\left(\mathrm{mg} / \mathrm{L}^{*} \mathrm{~h}\right)$ & 10.457 & 16.158 \\
MRT & 2.686 & 3.402 \\
Tmax $(\mathrm{h})$ & 0.083 & 0.083 \\
Cmax $(\mathrm{mg} / \mathrm{L})$ & 5.680 & 6.260 \\
\hline
\end{tabular}

less than $93 \%$. The mean drug concentration in plasma versus time profile after the intravenous administration of liposomes and suspension was shown in Figure 5, and the pharmacokinetic parameters calculated by DAS 2.0 software were summarized in Table 2. After i.v. administration, the plasma temozolomide concentration of temozolomide-liposomes group was much higher than that of the temozolomide solution group at each sampling time. Furthermore, temozolomideliposomes were eliminated more slowly than temozolomide solution. Besides, following injection of temozolomide-liposomes, temozolomide concentration was still measured to be $0.37 \mathrm{mg} / \mathrm{L}$ after $10 \mathrm{~h}$, while temozolomide concentration of temozolomide solution was detected to be $0.29 \mathrm{mg} / \mathrm{L}$ after $5 \mathrm{~h}$. Compared with temozolomide solution, the pharmacokinetic parameters of liposome group have been changed: $t_{1 / 2 \beta}$ and MRT were $13.312 \mathrm{~h}$ and $3.402 \mathrm{~h}$, which were 3.57 times and 1.27 times of temozolomide solution; $\mathrm{C}_{\max }$ and AUC values of temozolomide-liposomes were $6.26 \mathrm{mg} / \mathrm{L}$ and $16.158 \mathrm{mg} / \mathrm{L} \cdot \mathrm{h}$, which were 1.10 times and 1.55 times of temozolomide solution. The results of pharmacokinetics study showed that prolonged in vivo circulation time and increased AUC were in favor of temozolomide treatment for gliomas. These results could account for the rapid distribution and metabolism 

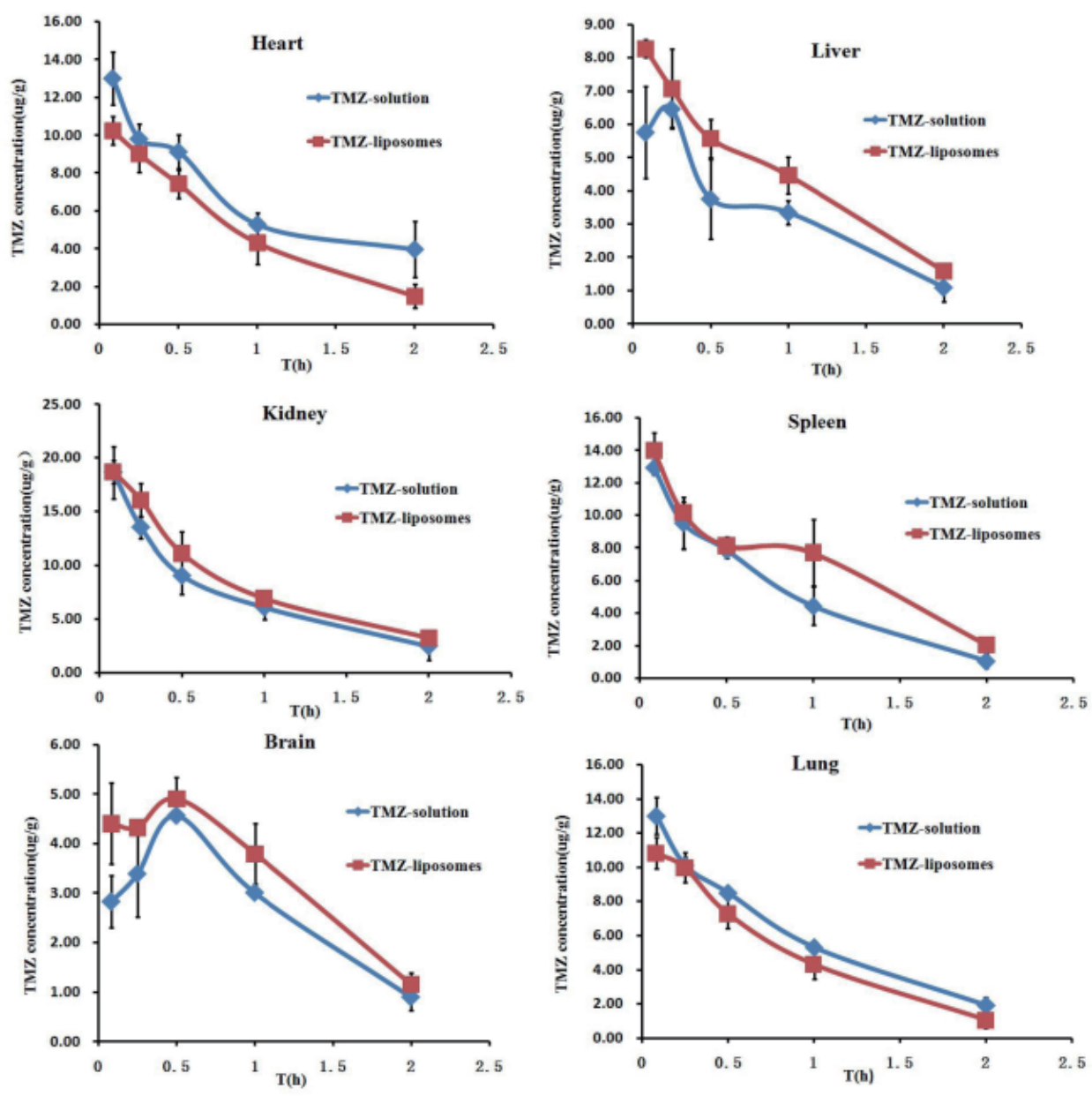

Figure 6. Temozolomide concentration-time curve in tissue at different time points after intravenous (i.v.) administration of temozolomide-solution and temozolomide-liposomes $(n=3)$. The biodistribution profiles of temozolomide in mice after intravenous administration of temozolomide solution and temozolomide-liposomes were shown. Briefly, the drug concentrations in brain were elevated after the administration of temozolomide-liposomes compared with the temozolomide solution. Meanwhile, the drug concentration in liver, kidney and spleen increased but decreased in heart and lung after administration of the liposomal formulation compared with temozolomide solution.

of the free temozolomide, compared to the distribution of temozolomide entrapped in liposomes. This might be explained by the protection of the lipid bilayer membranes and slow drug release from liposomes (18), which was also shown in the in vitro release experiment. At the same time a water shield on the surface of the liposomes reduced adsorption of opsonins, thereby reducing the intake of mononuclear phagocytes. That was one of the reasons why temozolomide-liposomes could remain in the circulation for a longer period of time than temozolomide solution.

\subsection{Drug distribution studies in mice}

The biodistribution profiles of temozolomide in mice after intravenous administration of temozolomide solution and temozolomide-liposomes were shown in Figure 6. Some changes of distribution can be observed between temozolomide solution and liposomes after the intravenous administration. Briefly, the drug concentrations in brain were elevated after the administration of temozolomide-liposomes compared with the temozolomide solution which could improve the efficiency of temozolomide treatment for gliomas. Meanwhile, the drug concentration in liver, kidney and spleen increased but decreased in heart and lung after administration of the liposomal formulation compared with temozolomide solution, which may be expected to reduce the potential side effects in heart and lung (19). The increasing concentration of temozolomide in liver and spleen may be explained by the fact that liposomes $<200 \mathrm{~nm}$ tend to be taken up by macrophages in liver and spleen more easily. The reason resulted to the increasing concentration of temozolomide in kidney was liposomes tend to be into the kidney easily due to the hydrophilic layer of sorbitol on the surface of liposomes (20). Furthermore, temozolomide-liposomes resulted in a higher drug accumulation in brain compared with temozolomide solution, which may be explained by the fact that the RES removal of the nanoparticles can be prevented by surface coating with hydrophilic polymers to increase their availability at brain (21). Liposome could be consumed by monocytes, neutrophils, etc., which can pass through blood brain barrier in the body 
Table 3. Tissue targeting parameters of temozolomide solution and temozolomide liposomes after $i . v$. administration in mice $(n=6)$

\begin{tabular}{llll}
\hline \multirow{2}{*}{ Tissues } & \multicolumn{2}{c}{ AUC } & \multirow{2}{*}{ Re } \\
\cline { 2 - 3 } & Temozolomide-Sol & Temozolomide-Lip & \\
\hline Heart & 13.58 & 10.366 & 0.763 \\
Liver & 6.812 & 9.106 & 1.337 \\
Spleen & 10.975 & 14.328 & 1.306 \\
Lung & 12.444 & 10.426 & 0.838 \\
Kidney & 15.178 & 17.494 & 1.153 \\
Brain & 5.656 & 6.9 & 1.220 \\
\hline
\end{tabular}

$\mathrm{Re}=$ AUCliposomes/AUCsolution.

circulation process selectively and get to the brain. Besides, it can get to the brain tissue through the blood brain barrier by pinocytosis of endothelial cells. So the drug concentration in the brain could increase significantly. At the same time a water shield on the surface of the liposomes reduce adsorption of opsonins, thereby reducing the passive targeting to lung.

To represent targeting efficiency, AUC and the targeting parameters such as the intake rate $(\mathrm{Re})$, were calculated and are listed in Table 3. temozolomideliposomes exhibited a larger value of AUC for brain compared with temozolomide solution. The results showed that compared to temozolomide solution, Re value of temozolomide-liposomes in the brain was 1.22 , Re values in the liver and spleen were 1.337 and 1.306, Re values in the kidney was 1.153 , and Re values in the heart, lungs were 0.763 and 0.838 , which also implied that temozolomide-liposomes increased the concentration of the drug in the brain, improved the therapeutic effect and reduced the toxicity in heart and lung.

In this study, temozolomide-liposomes were prepared for intravenous injection with the method of proliposomes successfully. The preparation process was simple with good reproducibility, and temozolomideliposomes possess displayed excellent performance featured by small and uniform size. The $\mathrm{pH}$ was within the safe $\mathrm{pH}$ scope of 4-9 for intravenous injection. The results of in vitro drug release behavior showed that temozolomide-liposomes released slowly compared with temozolomide solution. The results of pharmacokinetics and tissue distribution study showed that the liposomes had a slow-release effect and brain targeting. Furthermore, temozolomide-liposomes could reduce the $\mathrm{C}_{\max }$ in some organs such as heart, lung potentially decreasing the side effects. temozolomide-liposomes can change biodistribution, increase the concentration of drug at the treatment site, and reduce side effects in nontarget tissues. Furhermore temozolomide- liposomes avoided the use of Tween 80 which was contained in the listed freeze-dried powder for injection which might be helpful to decrease the side effects. The drug delivery system can prolong the half-life, improve the targeting and reduce the systemic effect of the drug, which were promising for developing the new formulation.
The following work would be focused on optimizing prescription and process to improve the encapsulation efficiency.

\section{Acknowledgements}

The authors wish to thank the School of Pharmaceutical Science, Shandong University, for supporting this study.

\section{References}

1. Ries L, Eisner M, Kosary C. SEER Cancer Statistics, 1973-1998. Bethesda, MD, National Cancer Institute. 2001; 2:1375-1382.

2. Galanis E, Buckner J. Chemotherapy for high-grade gliomas. Brit J Cancer. 2000; 82:1371.

3. Stupp R, Dietrich P-Y, Kraljevic SO, Pica A, Maillard I, Maeder P, Meuli R, Janzer R, Pizzolato G, Miralbell R. Promising survival for patients with newly diagnosed glioblastoma multiforme treated with concomitant radiation plus temozolomide followed by adjuvant temozolomide. J Clin Oncol. 2002; 20:1375-1382.

4. Baker SD, Wirth M, Statkevich P, Reidenberg P, Alton K, Sartorius SE, Dugan M, Cutler D, Batra V, Grochow LB. Absorption, metabolism, and excretion of 14C-temozolomide following oral administration to patients with advanced cancer. Clin Cancer Res. 1999; 5:309-317.

5. Kopecký J, Priester P, Slováček L, Petera J, Kopecký $\mathrm{O}$, Macingova Z . Aplastic anemia as a cause of death in a patient with glioblastoma multiforme treated with temozolomide. Strahlenther Onkol. 2010; 186:452-457.

6. Deng Y, Zhao J, Dong X, Shi L, Lu Y, Ni D, Zhao H. Composite emulsifier, an emulsion prepared from it and the preparation method thereof. U.S. Patent Application 12/670,820[P]. 2008-7-25.

7. Constantinides PP, Tustian A, Kessler DR. Tocol emulsions for drug solubilization and parenteral delivery. Adv Drug Deliver Rev. 2004; 56:1243-1255.

8. Huang GH, Zhang Z, Bi XL, Dou MJ. Solid lipid nanoparticles of temozolomide: Potential reduction of cardial and nephric toxicity. Int J Pharm. 2008; 355:314320.

9. Allen TM, Cullis PR. Liposomal drug delivery systems: From concept to clinical applications. Adv Drug Deliver Rev. 2013; 65:36-48.

10. Chonn A, Cullis PR. Recent advances in liposome technologies and their applications for systemic gene delivery. Adv Drug Deliver Rev. 1998; 30:73-83.

11. Afergan E, Epstein H, Dahan R, Koroukhov N, Rohekar K, Danenberg HD, Golomb G. Delivery of serotonin to the brain by monocytes following phagocytosis of liposomes. J Control Release. 2008; 132:84-90.

12. Garcia-Garcia E, Andrieux K, Gil S, Couvreur P. Colloidal carriers and blood-brain barrier (BBB) translocation: A way to deliver drugs to the brain? Int J Pharm. 2005; 298:274-292.

13. Huwyler J, Cerletti A, Fricker G, Eberle AN, Drewe J. By-passing of P-glycoprotein using immunoliposomes. J Drug Target. 2002; 10:73-79.

14. Hwang SY, Kim HK, Choo J, Seong GH, Hien TBD, Lee E. Effects of operating parameters on the efficiency of liposomal encapsulation of enzymes. Colloid Surface B. 
2012; 94:296-303.

15. Liu TS, Cai BC, Deng XK, Li WD, Zhao XH, Huang YZ. Preparation of strychnine solid liposome by sorbitol carrier aggradation and freeze-drying method. Chin Tradit Pat Med. 2005; 27:509-511.

16. Li BQ, Deng YJ, Yang JW. Determination of entrapment efficiency of topotecan liposomes by ultrafiltration-HPLC. Chin New Drug J. 2007; 16:58.

17. Lin QP, Guo RP, Wang QS, Wang DC, Liu CH, Xu $\mathrm{XY}$. Preparation, in vitro release and cytotoxic effect of docetaxel liposomes for intravenous injection. J Chin Pharm Univ. 2008; 39:417-421.

18. Ait-Oudhia S, Mager DE, Straubinger RM. Application of pharmacokinetic and pharmacodynamic analysis to the development of liposomal formulations for oncology.
Pharmaceutics. 2014; 6:137-174.

19. Maldonado F, Limper AH, Lim KG, Aubrey MC. Temozolomide-associated organizing pneumonitis. Mayo Clin Proc. 2007; 86:771-773.

20. Suzuki R, Takizawa T, Kuwata Y, Mutoh M, Ishiguro N, Utoguchi N, Shinohara A, Eriguchi M, Yanagie H, Maruyama K. Effective anti-tumor activity of oxaliplatin encapsulated in transferrin-PEG-liposome. Int J Pharm. 2008; 346:143-150.

21. Kaur IP, Bhandari R, Bhandari S, Kakkar V. Potential of solid lipid nanoparticles in brain targeting. J Control Release. 2008; 127:97-109.

(Received March 20, 2015; Revised April 14, 2015; Rerevised May 1, 2015; Accepted June 20, 2015) 